\title{
LA PRISE EN COMPTE DES RAPPORTS AU SAVOIR EN MÉDIATIQUE
}

\author{
Jack Guichard ${ }^{1}$
}

De l'enseignement à la diffusion des sciences... il y a plus d'un pas! Certains veulent penser la vulgarisation, l'exposition ou le multimédia scientifique, comme s'ils étaient seulement des moyens d'éducation mieux immergés dans la vie sociale et culturelle que l'école. En réalité, les formes médiatiques s'inscrivent dans d'autres institutions, suscitent des pratiques différentes, structurées par les modes de fonctionnement qu'imposent les spécificités du média. La différenciation entre savoirs formels et informels passe par l'analyse des différences entre les domaines scolaire et médiatique. Ainsi on se rend compte qu'on ne peut pas penser les visées d'une réalisation d'exposition (ou d'un multimédia de vulgarisation scientifique) en terme d'objectif (qui correspond à des critères de savoirs formels), mais en terme d'impacts souhaités.

Notre expérience de conception et d'exploitation de produits de médiation scientifique (en particulier à la Cité des Sciences et de l'Industrie et à l'Exploradome de Paris), ainsi que notre pratique de recherche sur la diffusion et l'éducation (IUFM et LIREST), nous permet de formaliser les interactions entre mise en scène et émergence des messages. Ainsi, on peut caractériser les procédés de transposition

\footnotetext{
1 Professeur des universités, IUFM de Paris et LIREST de Cachan.
} 
médiatique et d'induction du sens qui conduisent à des réalisations (en particulier dans le domaine des expositions scientifiques et des multimédias).

\section{La médiatique, un champ autonome par rapport au domaine scolaire}

Bien que le contexte général de l'usage des médias soit différent du cadre scolaire, les médias comme les expositions scientifiques "se donnent pour objectif de faire découvrir et de faire acquérir par un public une certaine quantité d'information sur un sujet donné. Ils se réfèrent toujours à un corpus de connaissances qu'ils ont la charge de diffuser"'. Il y a toutefois une différence importante entre les contextes formels et informels comme le montre le tableau ci-dessous.

\begin{tabular}{|c|c|c|}
\hline & $\begin{array}{c}\text { Contexte formel : } \\
\text { éducation "scolaire" }\end{array}$ & $\begin{array}{c}\text { Contexte informel : } \\
\text { culture "médiatique" }\end{array}$ \\
\hline codification & programmes & pas de projet à long terme \\
\hline public & groupe d'âge homogène & usagers diversifiés \\
\hline conditions & contraint & liberté de choix \\
\hline temps & scolaire & loisir \\
\hline organisation/temps & progression régulière & intermittent \\
\hline $\begin{array}{c}\text { évaluation pour l'usager } \\
\text { ou l'élève }\end{array}$ & système de validation & pas de système de validation \\
\hline enjeux & examens, contrôles & culture personnelle \\
\hline recherche de & réussite & plaisir et culture \\
\hline
\end{tabular}

\section{Le cadre "médiatique se différencie du cadre scolaire par de nombreux aspects}

D'abord, les moments et les lieux sont différents. Contrairement à l'école, où on dispense pour des groupes d'élèves d'âge homogène

1 B. SCHELE, Faire voir, faire savoir, la muséologie scientifique au présent, Québec, Musée de la civilisation, 1989. 
un enseignement réglementé par des instructions officielles, avec un programme et une progression bien définis, sanctionnés par des évaluations ou des examens, les médias scientifiques correspondent pour leurs usagers à une démarche volontaire, sur un temps de loisir, pratiquée de façon intermittente, sans système de validation. De plus, l'école est un espace clos où l'apprenant est mis en demeure d'apprendre ; elle a un pouvoir de coercition sur 1'attitude de réceptivité de l'apprenant (sanctions, diplômes) ; on y a du temps, c'est-àdire la possibilité de progression et de répétition. L'école a une finalité de recherche de réussite et de socialisation, qui n'a rien à voir avec la finalité ludique de l'espace culturel ${ }^{1}$. Par contre le contexte informel médiatique est un espace de liberté, que les usagers explorent avec des appuis et des projets différents les uns des autres. Chaque média n'a pas une place prédéfinie dans un projet d'éducation sur le long terme.

Ces différences entre l'éducation scolaire et les médias correspondent à des usages aux caractéristiques différentes. En particulier la relation aux médias se situe, pour leurs usagers, dans une perspective de recherche de plaisir et de culture personnelle. Cette culture scientifique médiatique correspond à une image de la science, à des activités scientifiques et à des connaissances dans ce domaine, produites et diffusées dans l'ensemble du public par les moyens de communication (expositions, revues, audiovisuels, télévisions, multimédias...).

Ces différences fondamentales conduisent souvent à opposer l'éducation scolaire, et ses savoirs "formels", et les médias, ces derniers véhiculant des savoirs "informels". Un des problèmes est que les médias sont aussi utilisés dans le cadre scolaire, et en ce qui concerne les expositions pour une part non négligeable des utilisateurs (puisqu'elle concerne de 40 à $60 \%$ de leurs visiteurs), ce qui n'est pas sans créer d'ambiguité.

\section{L'absence de feed-back direct entre le médiateur et son public}

Il existe une différence fondamentale entre la situation scolaire et la situation médiatique. Il n'y a pas, comme dans la situation scolaire, de feed-back direct entre le médiateur et les usagers. Tout passe par

1 E. CaIllet, "Culture scientifique, technique et industrielle", Éducation permanente, Paris, 1986. 
l'objet et la scène dans laquelle il se situe. L'usager du média construit du sens dans l'interaction avec le média. Cette absence de relation physique directe entre ces deux acteurs de la médiatique ne permet pas au concepteur, comme peut le faire l'enseignant, de "sentir" son public, de s'y adapter par des rétroactions successives et de modifier son action en fonction des réactions et du cheminement intellectuel de l'apprenant. En effet, si des obstacles apparaissent pour certains apprenants, l'enseignant peut modifier son discours, expliciter les informations, adapter son propos et son vocabulaire en faisant appel à d'autres schémas explicatifs. Le dispositif médiatique n'est pas modifiable lors de l'usage. Le concepteur a des visées, c'est-à-dire des idées sur ce qu'il souhaite obtenir comme effet sur les usagers du média. Il créé un dispositif médiatique, mais il ne s'adresse pas directement (physiquement) aux usagers. Le concepteur crée des objets, les met en situation, mais n'a pas d'interaction directe avec l'usager. Il choisit des messages qu'il met en objet, en scène ou en image en fonction de ses propres conceptions et parfois des recherches sur celle des usagers en tenant compte de leur diversité. Il ne dispose pas, comme l'enseignant, d'évaluation propre à chaque usager qui lui permette d'adapter son propos par la recherche de nouveaux moyens pour faciliter la compréhension. Il n'a donc aucune possibilité de régulation en cours d'usage du média. Il ne peut que constater le résultat.

Cette caractéristique a pour conséquence une amplification de l'importance des études préalables à la conception du produit, puisque ce dernier ne sera plus modifiable (ou difficilement). Cette situation implique donc d'autant plus la nécessité d'une prise en compte du point de vue du visiteur (que recherche t-il ?), de ses appuis et des obstacles (conceptions préalables, connaissances, intérêts, questions). Elle implique enfin d'envisager ce qu'il va faire, ressentir, comprendre, apprendre. Des maquettes ou des prototypes du dispositif médiatique permettent d'anticiper ces résultats et de modifier le produit pour sa réalisation définitive. Mais leur test ne correspond jamais exactement aux conditions d'utilisations variées du produit final. Une fois le média disponible pour l'usager, il est aussi possible de mettre en place des études pour savoir si les impacts envisagés sont atteints ou non, pour quel type de visiteur et dans quelles conditions. 


\section{L'appropriation par les publics : obstacles et appuis}

La conception d'un dispositif médiatique porte sur : les contenus, les objets et les situations. Afin d'inventer des dispositifs médiatiques adéquats, ou d'adapter ses visées, on cherche à connaître les utilisateurs.

\section{Prise en compte des connaissances mobilisables par les utilisateurs des produits de vulgarisation}

Connaître les centres d'intérêt, les attentes, les conceptions, le cadre de référence des utilisateurs des produits que l'on crée est un préalable important. Cette approche est indispensable afin d'apporter des éléments de réponse aux questions du concepteur : qui sont les utilisateurs de mon produit? Qu'est ce qui va et comment les intéresser ? Quelles sont leurs questions, leurs attentes ? Quelles sont leurs façons de raisonner, leurs cadres de références, leurs façons de produire du sens ? Pourront-ils lire le message écrit ? Comment puisje leur éviter tel obstacle ? Puis-je les aider à le dépasser?

De nombreux études ${ }^{1}$, établissent un constat sur les conceptions des apprenants et montrent l'importance de leur prise en compte dans l'enseignement. Chacun a des idées préconçues et interprète les phénomènes qu'il observe au travers de leur propre cadre de référence. De nombreux exemples ont démontré l'intérêt de cette prise en compte et en particulier lorsque les conceptions sont des obstacles pour la compréhension des phénomènes ${ }^{2}$. A partir de cette connaissance des questions et des connaissances des jeunes, on peut limiter le décalage fréquent entre les jeunes et le médiateur, mieux cibler les impacts à atteindre, trouver les situations et les arguments pour convaincre l'utilisateur, définir sur un plan pratique le point d'accroche du propos que l'on veut communiquer, le niveau conceptuel auquel il faut se placer ou le type de langage à utiliser.

1 A. GIORdAn, G. DE VECCHI, Comment faire pour que ça marche, Paris, Delagrave, 2000.

2 J. Guichard, F. Guichard, "Des objets muséologiques pour aider à traiter des obstacles en Sciences et Techniques", Aster, n² 24, Paris, 1997, pp. 113-141. 
La connaissance des conceptions des visiteurs peut permettre de définir les obstacles à faire franchir aux visiteurs. Ainsi nous avons conçu une manipulation sur "les muscles du bras" qui conduit l'enfant à remettre en cause ses idées sur la relation muscles-squelette. L'enfant doit trouver, par essai-erreur à quel endroit accrocher le biceps pour faire se plier le coude. Elle a été conçue en partant des résultats d'une enquête qui a montré l'absence de conception opérationnelle pour comprendre le rôle des muscles dans le mouvement des membres : $95 \%$ des utilisateurs ne représentent pas un muscle attaché sur 2 os différents de part et d'autre d'une articulation. Les résultats de cette manipulation montrent que plus de la moitié des enfants commencent par placer le muscle de façon incorrecte ; ils modifient alors spontanément le point d'accroche pour un second essai, cette fois concluant. La manipulation induit donc une analyse du système muscle-articulation et une réflexion sur son fonctionnement. Un posttest montre que pratiquement tous les enfants qui effectuent cette manipulation assimilent le principe et le rôle du muscle dans le mouvement du bras.

\section{Une approche systémique et multi-référentielle}

Chaque usager s'approprie le média en intégrant les données qu'il perçoit à son propre système de représentation. Certains recherchent d'abord un moment de loisir, d'autres à acquérir une culture scientifique, d'autres enfin viennent étudier un sujet scientifique. Leurs visées sont donc bien différentes les unes des autres. Le constat de la diversité des usages des médias conduit leurs concepteurs à multiplier les types de situations dans les expositions, à multiplier les entrées et les hyperliens dans les multimédias, afin de proposer des utilisations différentes selon les utilisateurs du produit, avec une liberté de choix, donc de progression dans l'exposition ou le multimédia.

\section{Prendre en compte les pratiques familières des utilisateurs}

La conception d'un dispositif médiatique ne se limite pas au texte du savoir, le poids de la mise en scène est indissociable du choix des contenus. Parmi les appuis et les obstacles à prendre en compte lors de la création d'exposition ou de multimédias, les pratiques fami- 
lières sont fondamentales. Les enfants en particulier ont des rapports aux objets et aux situations bien différents de ceux des adultes ${ }^{1}$. Aussi, il convient de prendre en compte les réactions spontanées des jeunes en face d'un bouton, d'une manivelle, d'un flacon à sentir, d'une loupe à utiliser... mais aussi leur perception de l'écran ou du modèle que vous leur présentez et qui est en décalage avec la réalité qu'ils connaissent. Ce lien entre modèle et réalité ne peut s'effectuer que si cela a été pris en compte dans l'élaboration même du modèle, voire dans sa confrontation avec la réalité2.

Ainsi dans l'exposition "Technocité" la modification des boutons, sur lesquels il faut appuyer pour déclencher un phénomène à étudier, a changé le comportement des jeunes. Alors que face à des gros boutons ils tapaient avec le poing sans observer le résultat de leur action, face à des petits interrupteurs enfoncé dans la table, ils ne peuvent appuyer qu'avec l'index, ce qui focalise leur attention de longues minutes et leur permet de découvrir tout l'intérêt de l'exhibit. Les enfants cherchent l'action, la surprise. Ils ne résistent pas au plaisir d'aller chercher ce qui est caché : des textes cachés sous des trappes, ou sur des tirettes, des plaques à soulever. Leur curiosité ne résiste pas à une question courte et bien visible sur une trappe : ils soulèvent pour voir ce qui est en dessous.

La prise en compte de ces pratiques, en particulier pour l'ergonomie des écrans, a aussi son importance pour les créateurs de multimédias et de sites Internet. Ainsi il n'est pas si facile de présenter les mots ou les icônes qui correspondent à des hyperliens, ou les barres de menus de façon qu'elles soient compréhensibles par tous, surtout par les adultes non-initiés.

\section{Les dispositifs peuvent créer des obstacles à la compréhension}

La mise en scène ou en page de l'objet scientifique a aussi son importance : en effet il s'agit toujours d'une recomposition où l'ambiance qui est créée a une importance déterminante. Seuls des

1 S. Girardet, C. Merleau-PontI, Portes ouvertes : les enfants, Accueillir les enfants dans un musée ou une exposition, Dijon, OCIM, coll. "Expo mode d'emploi", 1994.

2 J. Guichard, "Adapter la muséologie aux enfants", in B. SCHIEle, E. Koster, La révolution de la muséologie des sciences : vers les musées $d u X X I^{2 m e}$ siècle, Lyon, Éditons Multimondes, Presses Universitaires de Lyon, 1998, pp. 207-248. 
tests de prototype nous ont permis de découvrir que la présentation d'une fourmilière de laboratoire ne permettait pas aux visiteurs de comprendre la disposition et le fonctionnement de la fourmilière car son aspect artificiel et sa disposition les amenait même à se construire des représentations erronées de la structure de l'objet dans la nature.

\section{Impact ou effets recherchés}

La prise en compte des obstacles et des appuis dans l'appropriation par les publics, associée à l'analyse des caractéristiques de la transposition médiatique, conduisent à définir un état des lieux (des usagers du média) et à avoir des visées (pour le média). Même pour les scolaires qui visitent une exposition ou consultent un multimédia ou une revue, l'enseignant n'assure pas sur place ses rapports au pouvoir et au savoir, ces derniers étant délégués au média. La situation est donc, de ce point de vue, bien différente de la situation scolaire. Elle amène à concevoir des zones d'incertitude à la fois par rapport au contenu de savoir, mais aussi par rapport aux publics cibles. Ce qui ne veut pas dire que les médiateurs ne savent pas ce que doit être le contenu, mais qu'ils ne peuvent prétendre avoir tout contrôle sur lui. Tout le travail du médiateur va donc consister à chercher des outils pour réduire cette part d'incertitude. Pour cela, dans le cadre scolaire, l'enseignant se fixe des objectifs, dont la définition est un enjeu important dans leur pratique pédagogique. Mais qu'en est-il en médiatique?

Il est possible de connaître les intentions et les motivations de l'usager du média: ce qu'il voudrait connaître, le plaisir qu'il recherche, ses savoirs préexistants. Les résultats de nos analyses d'expositions viennent confirmer l'analyse de J. L. Martinand ${ }^{1}$ sur les conditions de l'émergence du sens dans l'exposition. Le visiteur ne cherche pas directement des messages et un discours, mais parcourt un ensemble d'éléments dont il attend des informations et du plaisir sur des questionnements qui lui sont propres. En effet, le visiteur s'appuie sur son expérience ${ }^{2}$. Il est donc fort utile d'essayer de la

1 J.-L. MARTinAND, "Questions actuelles de la didactique des sciences", in A. GIORDAN, VINH BANG, Psychologie génétique et didactique des sciences, Berne, Peter Lang, 1989, pp. 93-104.

2 J.-P. NATAli, J.-L. Martinand, "Une exposition scientifique thématique... est-ce bien concevable ?", Education permanente, n 90, 1987. 
cerner, en référence au message que souhaite transmettre le scientifique, afin de définir les impacts recherchés. L'idée d'impact rejoint celle d'objectif-possible de Martinand'. Il ne s'agit pas seulement d'étudier les idées des usagers, mais de prendre conscience des obstacles à franchir dans le but de centrer les visées sur ces obstacles. La stratégie du concepteur vise donc à créer des dispositifs muséographiques et des conditions (situations, ambiances) induisant chez le visiteur les impacts désirés : impacts en termes d'émergence de sens, d'induction d'attitudes, de démarches, de connaissances.

La notion d'impact médiatique prend en compte la séparation physique du concepteur du produit et de ses usagers au moment de l'utilisation du produit, qui n'est alors plus modifiable. Elle conduit à s'intéresser au point de vue du concepteur et au point de vue de l'usager au moment de la conception du produit.

Les impacts recherchés pour le média correspondent aux effets que l'on cherche à provoquer. Dans le cas des médias, et en particulier des expositions, on ne peut pas isoler les impacts intellectuels (comprendre, analyser, mémoriser,...), du psychomoteur (explorer physiquement, manipuler, agir...) et du socio-affectif (choisir, ressentir, être ému, curieux, sensible à la beauté... $)^{2}$. Mais tous ces éléments ne sont pas si facilement évaluables.

\section{La recherche d'impacts passe par une évaluation prédictive avec tests de maquettes et de prototypes}

C'est par l'observation des comportements des usagers face à des prototypes et de maquettes des exhibits ou des multimédias, que le médiateur peut adapter ses créations en fonction de ses visées.

Dans l'élément d'exposition de la cité des sciences, "voir à l'intérieur du corps", l'image de l'intérieur du corps est reconstituée en image de synthèse. Des tests d'images avec des enfants ont démontré que le seul type d'image compréhensible était la radiographie colorée par ordinateur et animée. Une petite animation par ordinateur, montra la nécessité de ne pas représenter le trajet de l'air en bleuté, car les enfants l'assimilaient à du liquide et non à de l'air ; d'autre

1 J.-L. MARTINAND, Les recherches en didactique des sciences et de la technologie, conférence, Paris, Centre IDEN, 1989.

2 L. Legrand, Pour une pédagogie de l'étonnement, Neuchâtel, Delachaux et Niestlé, 1988. 
part elle fit ressortir également le besoin de donner du volume à l'image pour augmenter le réalisme et la compréhension, qui fut réalisé en image de synthèse.

Ainsi le test de prototype permet d'anticiper les réactions des visiteurs ou des utilisateurs. Il évite bien des erreurs dans la conception des produits de vulgarisation. La recherche de l'impact est, pour le concepteur, un outil pragmatique pour une prise de décision.

\section{Rapport au savoir}

La question de l'impact des réalisations dans ces domaines est un souci important des réalisateurs et surtout des pédagogues qui souhaitent les utiliser avec leurs élèves. Mais souvent on assiste à une incompréhension entre les scientifiques et les réalisateurs des produits. Les scientifiques cherchent à transmettre des connaissances. Les réalisateurs cherchent d'abord à faire un produit attractif qui a du succès. Or le public est d'abord sensible à cette mise en scène et ne perçoit pas toujours le savoir qu'on cherche à transmettre. Face à ce fréquent problème, des scientifiques considèrent alors le produit comme un échec dû aux réalisateurs qui n'ont pas voulu les écouter. Les réalisateurs répondent que le produit n'est pas fait pour transmettre des connaissances, mais pour le plaisir ou la délectation esthétique. Cette fréquente incompréhension entre scientifiques et créateurs peut-être supprimée si on évalue l'impact du produit en terme de rapport au savoir et non en terme de connaissances acquises par le visiteur.

Lors de la visite d'une exposition, comme lors de l'utilisation d'un multimédia ou d'une revue de vulgarisation sur son temps de loisir, le rapport au savoir est très différent de celui des élèves dans la classe. Lors d'une visite d'exposition (exhibition), par exemple, le visiteur ne se contente pas d'écouter et de faire ce que demande l'enseignant. Il est immergé dans un environnement qu'il parcourt avec ses pieds, perçoit avec tous ses sens, et sur lequel il agit parfois avec ses mains. Il y recherche avant tout le plaisir de la découverte. Il est libre de se déplacer et d'agir.

Le concepteur de produits de vulgarisation ne peut donc pas se contenter de prendre en compte le rapport des utilisateurs aux informations exposées. En effet ce n'est pas qu'un problème de connaissances scientifiques, mais celui du rapport au savoir des utilisateurs 
du produit. Du point de vue des savoirs, il ne s'agit pas seulement de leur rapport aux connaissances, mais aussi de leur image de la science et des modalités d'accès à ce savoir. Le rapport au savoir à prendre en compte comporte aussi une dimension affective de désir, de plaisir, d'émotion, d'intérêt développé par le média. Le contexte y a une importance fondamentale, car il détermine les conditions pour l'émergence de sens, au travers de la contextualisation des savoirs ou de la mobilisation pour l'activité dans une exposition interactive'. Leur rapport au savoir est à la fois psychologique, physique et intellectuel.

Le concepteur des produits de vulgarisation ne doit donc pas seulement se soucier du texte du savoir, mais d'abord donner envie de découvrir les informations. Il doit aussi tenir compte de la façon d'investir les lieux ou les dispositifs proposés. De plus pour la conception de ces produits, il est nécessaire de prendre en compte la diversité des publics et de leurs cheminements intellectuels.

Le besoin de connaître les phénomènes sur lesquels porte l'évaluation dans le musée explique le retour incessant que l'on observe dans les publications et les colloques sur l'objet de l'évaluation et sur les instruments à utiliser, alors que tous deux sont clairement établis ${ }^{2}$. Les concepteurs d'exposition sont constamment confrontés à la question de l'impact de leurs actions, certains préférant se contenter de considérer l'exposition comme un lieu uniquement de délectation, alors que beaucoup de scientifiques la considèrent comme un "lieu d'éducation scientifique approfondie". Le concept de rapport au savoir dans l'exposition peut sans doute paraître paradoxal aux protagonistes de ces deux attitudes contradictoires, mais il permet de poser le problème de façon plus rationnelle.

\section{Le rapport au savoir en médiatique}

Dès que l'on parle, par exemple de lutte contre l'échec scolaire, on s'aperçoit que c'est la question du rapport au savoir qui est opérationnelle. Comme le définit B. Charlot "le rapport au savoir est le

1 J. Gutchard, J.-L. Martinand, Médiatique des sciences, Paris, PUF, coll. "Éducation et de formation", 2000.

2 C. DUFrESNE-TASSE, "Comparaison du rôle de l'évaluation à l'école et au musée : implication pour la pratique muséale", in M. ALLARD, B. LEFEBVRE, Le Musée, un lieu éducatif, Québec, Musée d'art contemporain de Montréal, 1997, pp. 353-361.

3 B. Charlot, Du rapport au savoir, éléments pour une théorie, Paris, Anthropos, 1997. 
rapport au monde, à l'autre et à soi-même, d'un sujet confronté à la nécessité d'apprendre. Le rapport au savoir est l'ensemble organisé des relations qu'un sujet entretient avec tout ce qui relève de l'apprendre et du savoir". Pour Lenoir ${ }^{1}$, il faut entendre par rapport au savoir, "à la fois les représentations de ce qu'est le savoir et des modalités d'accession à ce savoir", c'est-à-dire le processus d'objectivation par lequel l'individu établit un rapport au réel et donne du sens à la réalité qu'il conceptualise.

Considérer le rapport au savoir conduit à s'intéresser au processus qui conduit à l'adopter et pas simplement au contenu intellectuel du savoir. Le rapport au savoir, en médiatique, inclut l'idée de mobilisation de l'usager dans son action. Sa constitution est "source de la capacité de penser et d'apprendre"2. Il englobe les savoirs d'expérience, c'est-à-dire l'ensemble des savoirs acquis et requis pour effectuer des activités, savoirs pratiques qu'il est utile de connaître pour définir les stratégies à mettre en ouvre dans les expositions ou les multimédias.

Pour Charlot, "le concept de rapport au savoir implique celui de désir"3. Du point de vue des médias, dire qu'un dispositif muséographique ou médiatique a du sens, ne veut pas simplement dire qu'il a de la signifiance, mais veut aussi dire qu'il peut provoquer du désir, mobiliser, mettre en mouvement un usager qui lui trouve de la valeur. Cet aspect de la question est fondamental en médiatique, où il faut d'abord capter l'intérêt de son public en déclenchant son désir.

Pour Beillerot, l'accès à un nouveau savoir est l'accès à un objet extérieur au sujet. Son appropriation est une re-création de l'objet dans le sujet apprenant ${ }^{4}$. On ne comprend pas, si on n'est pas capable de saisir le caractère suivi du discours. Pour comprendre, on doit pouvoir trouver le sens global, la cohérence, c'est-à-dire les liens qui existent à l'intérieur d'un discours entre des éléments constitutifs (cohérence interne d'un texte) et entre ce discours et le contexte dans lequel il se situe (cohérence externe). La contextualisation des savoirs est donc un facteur essentiel lorsqu'on s'adresse à des usagers de média en dehors de tout curriculum. La compréhension est un ajuste-

1 Y. LENOrR, "Médiation cognitive et médiation didactique", in De la médiation didactique, 1998, pp. 223-251.

2 J. Bellerot, C. Blanchard-Laville, N. Mosconi, Pour une clinique du rapport au savoir, Paris, L'Harmattan, 1996.

3 B. CHARLOT, op. cit.

4 J. BELlerot, C. Blanchard-LAville, N. MosCon, op, cit. 
ment permanent entre un donné et les connaissances que le sujet mobilise pour comprendre. On comprend donc pourquoi il est important de connaître les appuis des usagers du média.

\section{Le rapport à l'ignorance}

Il est intéressant de poser la question du rapport au savoir en médiatique en la liant à celle du rapport à l'ignorance. Des études menées sur une exposition sur le thème de l'informatique ${ }^{1}$ ont montré que la majorité des visiteurs "loin de se constituer spontanément en cible d'une initiative pédagogique destinée à leur faire acquérir des connaissances dans ce domaine, affirment spontanément leur droit à l'ignorance et revendiquent la légitimation d'un savoir d'usagers, un savoir d'ignorants, par opposition à un savoir de spécialistes". Dans ce cas, le thème de l'exposition recouvrait pour eux des enjeux sociaux qui sont beaucoup plus importants que les connaissances savantes sur le domaine. Cette approche est fondamentale pour comprendre les attentes du visiteur de l'exposition et envisager les impacts possibles. Chacun y développe son propre rapport au savoir, qui n'est ni celui du scientifique, ni celui du médiateur, ni celui du scénographe, ni celui du visiteur voisin! D'où l'importance de prendre en compte l'extrême diversité des visiteurs de l'exposition et pas simplement "un visiteur moyen". De même pour les autres médias.

Il est donc intéressant de penser l'ignorance de façon positive. Personne ne peut, ni tout connaître, ni s'intéresser à tout. Tout individu s'intéresse à des choses et refuse de s'intéresser à d'autres. Dans le média, on choisit ce qui correspond aux savoirs que l'on veut développer en écartant les autres éléments ou en les considérant seulement d'un point de vue artistique ou affectif. Le média, qui veut toucher un large public, n'est pas conçu que pour apporter des savoirs, mais aussi pour procurer du plaisir, de l'émotion... L'ignorance est nécessaire à la diffusion des sciences. En effet l'ignorance qu'une situation de recherche permet d'objectiver est "une source d'émerveillement proportionnelle à cette objectivation" 2 . La diffusion des

1 J. LE MAREC, "Repenser la relation du musée avec son public", in La révolution dans la muséologie des sciences, Lyon, Multimondes, 1998, pp. 379-396.

2 T. IVAINER, R. LENGLET, Les ignorances des savants, Maison Neuve et Larose éd., 1996. 
connaissances doit faire partager les interrogations, les doutes des scientifiques et les mystères avant de trouver les éléments de réponse. L'ignorance donne naissance à des étonnements, prend la forme de doutes et de problèmes. Que de situations médiatiques peuvent s'appuyer sur cette caractéristique !

Le rapport à l'ignorance est aussi un concept intéressant car, dans une démocratie, l'ignorant peut aussi exiger des scientifiques une véritable recherche de vulgarisation.

\section{Conclusion}

La prise en compte des rapports au savoir conduit à inventer des objets qui peuvent, par leur impact émotionnel ou les stratégies interactives qu'ils proposent, mettre les usagers des médias en situation de remettre en cause leurs conceptions. Ce type de situation peut être développé si les vulgarisateurs s'appuient sur une bonne connaissance de ces obstacles.

Contrairement aux savoirs formels construits dans le cadre d'un curriculum et d'une progression, les savoirs informels ne peuvent pas être simplement pensés comme une construction de connaissances scientifiques.

Dans la vulgarisation, le rapport au savoir n'est pas qu'un rapport au savoir savant, mais prend des dimensions beaucoup plus larges. "Le message vulgarisateur n'est pas un message lacunaire dont il suffirait de corriger les imperfections et les erreurs pour le faire accéder au statut du message scientifique ou didactique"1. Cette approche diffère de l'idée négative qu'ont certains scientifiques de la vulgarisation en la pensant comme une perte de connaissance ${ }^{2}$, une simplification de la connaissance scientifique.

1 B. SCHIEle, G. LARocQue, "Le message vulgarisateur", Communication : Apprendre des médias, $\mathrm{n}^{\circ} 33,1981, \mathrm{p} .168$.

2 Idée qui renforce d'ailleurs leur pouvoir, alors que la démocratie devrait exiger d'eux cet effort de vulgarisation. 